\title{
Temperature dependent device characteristics of organic light-emitting devices
}

\author{
Stefan Berleb *, Anton G. Mückl, Wolfgang Brütting, Markus Schwoerer \\ University of Bayreuth, Experimental Physics II, 95440 Bayreuth, Germany
}

\begin{abstract}
Current-voltage characteristics of single and hetero-layer light-emitting devices with an aromatic diamine (TPD) as hole transport material and tris-8-(hydroxyquinoline) aluminum $\left(\mathrm{Alq}_{3}\right)$ as electron transport material and emitter have been investigated over a wide temperature range and for various film thickness in order to identify the limiting mechanism: charge carrier transport or injection. From the observed thickness and temperature dependence, pure injection limitation can be ruled out as dominant mechanism. Instead, the voltage dependence of the current density can be well described by power laws $j \propto V^{m+1}$ (with $V$ corrected by the built-in potential) with temperature dependent exponents $m$ ranging from 4 to 25 . This can be interpreted in terms of space charge limited currents (SCLC) in $\mathrm{Alq}_{3}$ with an exponential energetic distribution of traps where $m$ is given by $m=E_{\mathrm{t}} / k T$. A reasonable trap energy of $0.15-0.2 \mathrm{eV}$ is obtained by a temperature dependent analysis of the $I-V$ characteristics. However, the thickness dependence cannot be satisfactorily explained by the simple SCLC-model. This indicates that more sophisticated models are required.
\end{abstract}

Keywords: Light-emitting devices; Current-voltage characteristics; Temperature-dependence

\section{Introduction}

The field of organic electroluminescence has gained growing interest in recent years in scientific and industrial research due to a high potential for display applications. Though the phenomenon itself had been known for a long time [1] this rapid development was initiated by the publication of Tang and Van Slyke in 1987 [2]. By using a hetero-structure device they could improve the performance remarkably. This so-called 'Kodak-structure' consisted of an aromatic diamine (TPD) and a metal-chelate complex $\left(\mathrm{Alq}_{3}\right)$. As many research groups are working with this or similar structures, it can be regarded as a 'model device' for organic light-emitting devices based on small molecules. Although much work has been done to explain the electro-optical characteristics of such devices, there still remain many open questions concerning charge carrier transport and injection. Different experimental techniques covering a wide range of device parameters for the same materials are necessary to obtain further insight. Therefore, we have measured the characteristics of single

\footnotetext{
* Corresponding author.

E-mail address: stefan.berleb@uni-bayreuth.de (S. Berleb).
}

and hetero-layer devices based on $\mathrm{Alq}_{3}$ and TPD as a function of temperature and organic layer thickness and compared the experimental findings with the theoretical predictions of different models which are commonly used in the context of organic light-emitting devices.

\section{Experimental setup and results}

In all devices under investigation the organic layers were sandwiched between ITO and $\mathrm{Ca}$ electrodes. A positive voltage (forward bias) implies that the ITO electrode is positively biased with respect to the $\mathrm{Ca}$ electrode. The active area was $4 \mathrm{~mm}^{2}$ for the single layer devices and 14 $\mathrm{mm}^{2}$ for the double layer devices. All measurements were carried out under nitrogen atmosphere in a liquid nitrogen cryostat. Current-voltage characteristics were measured with a Keithley source-measure unit 237. Electroluminescence intensity was obtained by simultaneously recording the current of a calibrated photodiode with an electrometer.

In the analysis of the current-voltage characteristics one has to take into account the built-in potential when electrodes with different work-functions are used. The built-in potential $V_{\mathrm{bi}}$ is approximately equal to the difference of the work-functions and reduces the externally 
applied voltage. The consequence of the built-in potential is that there exists a non-vanishing electric field in the organic layer(s) already for zero applied bias, which has to be overcome before a forward drift current can be obtained. From our $I-V$-measurements at room-temperature on single and hetero-layer devices (see below), an upper limit is estimated from the current onset to about $2-2.2 \mathrm{~V}$ (Fig. 1), while a lower limit is estimated from the open-circuit voltage under strong illumination to $1.8 \mathrm{eV}$. Therefore, for the following analysis a built-in potential of $1.9 \mathrm{eV}$ is used. With respect to transport or injection models the built-in potential can be included by changing the boundary condition for the electric field $F$ to $\int_{0}^{1} F d x=V-V_{\mathrm{bi}}$. If a spatially homogeneous electric field is assumed within the device this leads to $F=\left(V-V_{\mathrm{bi}}\right) / d$ where $d$ is the organic layer thickness.

The determination of the electric field distribution in a hetero-layer device is not straightforward as accumulated charges, e.g., at interfacial barriers or injecting contacts, will lead to local changes in the electric field. However, qualitative insight can be obtained by comparing the thickness and voltage dependence of the current for the materials under investigation in single layer configuration. In Fig. 1 the current-voltage characteristics for TPD and $\mathrm{Alq}_{3}$ single layer devices are depicted for different organic layer thickness. All devices exhibit a sharp increase of the current above $2-2.2 \mathrm{~V}$, which is less pronounced for the $\mathrm{Alq}_{3}$ devices. This indicates the upper limit of the built-in potential. The current for the TPD devices is much higher for a given voltage and film thickness than for the $\mathrm{Alq}_{3}$ devices. It almost reaches the theoretical limit for trap-free space charge limited currents, indicating that our ITO forms a good hole injecting contact with TPD. The $I-V$ characteristics of the $\mathrm{Alq}_{3}$ single layer devices show a very pronounced thickness dependence in contrast to the TPD devices. Due to the differences between these two materials in a hetero-layer device where the electron current and the hole current are relatively balanced, a much

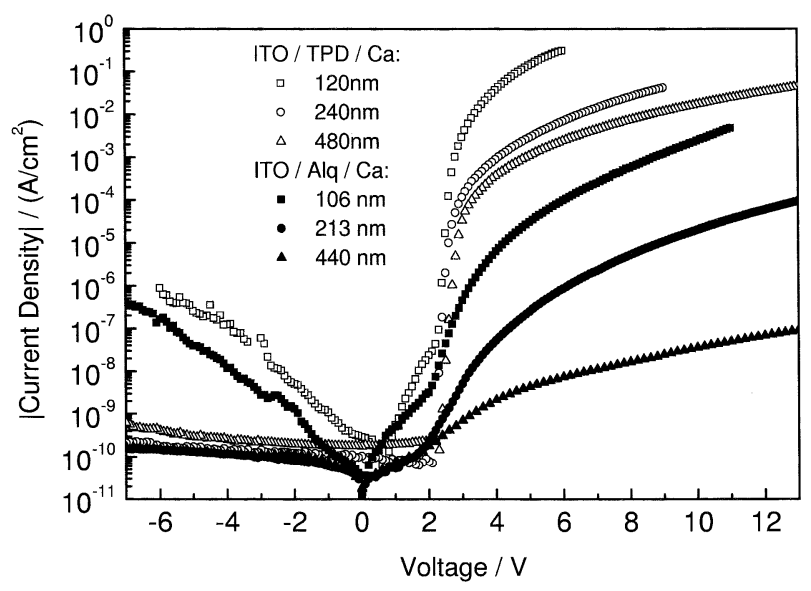

Fig. 1. Current-voltage characteristics of TPD and $\mathrm{Alq}_{3}$ single layer devices with an ITO anode and a Ca cathode for different film thickness.

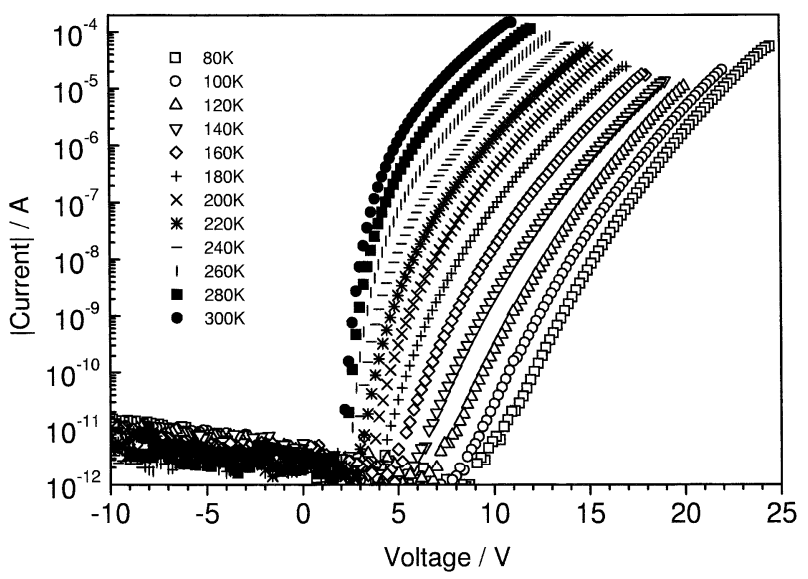

Fig. 2. Temperature dependent $I-V$-characteristics of an ITO $/ \mathrm{Alq}_{3}[106$ $\mathrm{nm}] / \mathrm{Ca}$ device in the range from 80 to $300 \mathrm{~K}$.

lower field is required to obtain the same current flow in the TPD layer as compared to the $\mathrm{Alq}_{3}$ layer. Hence, in such a device the voltage will drop mostly over the $\mathrm{Alq}_{3}$ layer. Therefore, we have concentrated our investigations mainly on $\mathrm{Alq}_{3}$ single layer devices.

Typical temperature dependent $I-V$-characteristics of an ITO $/ \mathrm{Alq}_{3} / \mathrm{Ca}$ device with an organic layer thickness of $106 \mathrm{~nm}$ are shown in Fig. 2. The current is strongly temperature dependent and varies, e.g., from $90 \mu \mathrm{A}$ at 10 $\mathrm{V}$ at room temperature to $5.7 \mathrm{pA}$ at $80 \mathrm{~K}$. To obtain the same current much higher voltages are needed at lower temperatures as compared to room temperature. The brightness-voltage characteristics (not depicted here) show a similar behaviour. The quantum efficiency rises from $0.1 \%$ at $300 \mathrm{~K}$ to $0.7 \%$ at $80 \mathrm{~K}$. Similar results are obtained for different values of $\mathrm{Alq}_{3}$ layer thickness $d$ $(213,330$ and $550 \mathrm{~nm})$.

In the case of the hetero-structure $\mathrm{TPD} / \mathrm{Alq}_{3}$, also a pronounced temperature dependence of current and brightness can be observed (see also Fig. 5). In contrast to the single layer device the quantum efficiency increases only slightly from $1.2 \%$ at $300 \mathrm{~K}$ to $1.6 \%$ at $140 \mathrm{~K}$ and drops again to the room temperature value for $T=80 \mathrm{~K}$.

\section{Discussion}

We will now compare the experimental findings with different models which are commonly used in the context of organic LEDs. They can be divided into two categories: bulk-limited and injection-limited. Typical models for injection-limitation are thermionic emission ('RichardsonSchottky') and tunneling through a triangular potential barrier ('Fowler-Nordheim'). Both models assume that the current is solely limited by high injection barriers at the electrodes - typically some tenths of an eV - implying that no space charges are present and therefore the electric field is homogeneous within the device. Hence, the current 


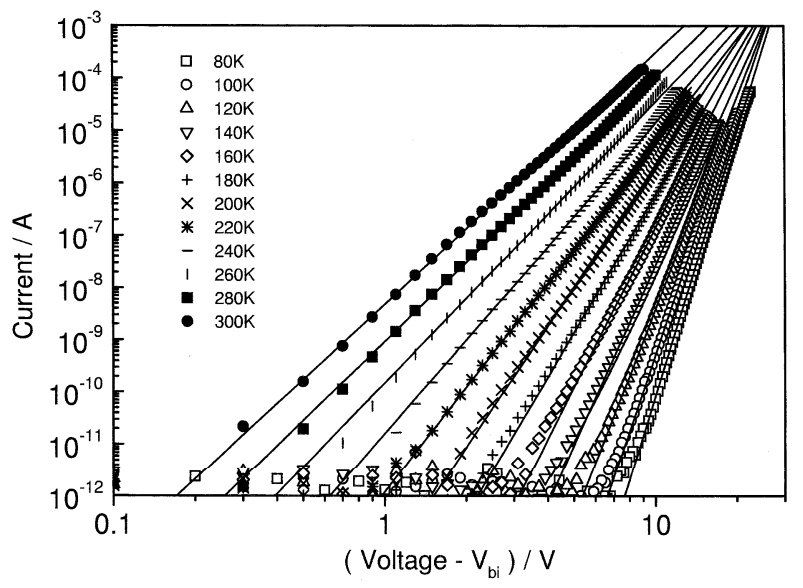

Fig. 3. Double-logarithmic representation of the temperature-dependent $I-V$-characteristics of an ITO/ $\mathrm{Alq}_{3}[106 \mathrm{~nm}] / \mathrm{Ca}$ device. The voltage was corrected by the built-in potential. The straight lines represent fits according to Eq. (1).

can be written as a function of the electric field alone without an explicit dependence on the device thickness. In other words, for a given electric field the current should be independent of the film thickness. However, in the $\mathrm{Alq}_{3}$ single layer devices the current for a given field decreases by about 2-3 orders of magnitude when increasing the film thickness from 100 to $550 \mathrm{~nm}$. This clearly rules out theoretical models based on pure injection limitation.

The strong variation of the current with the organic layer thickness for given fields can only be explained by a locally inhomogeneous electric field implying the presence of space charges. For materials with low carrier mobilities the current flow in the case of bulk-limitation can be described by the space charge limited current (SCLC) model. One requirement for SCLC is that enough charge

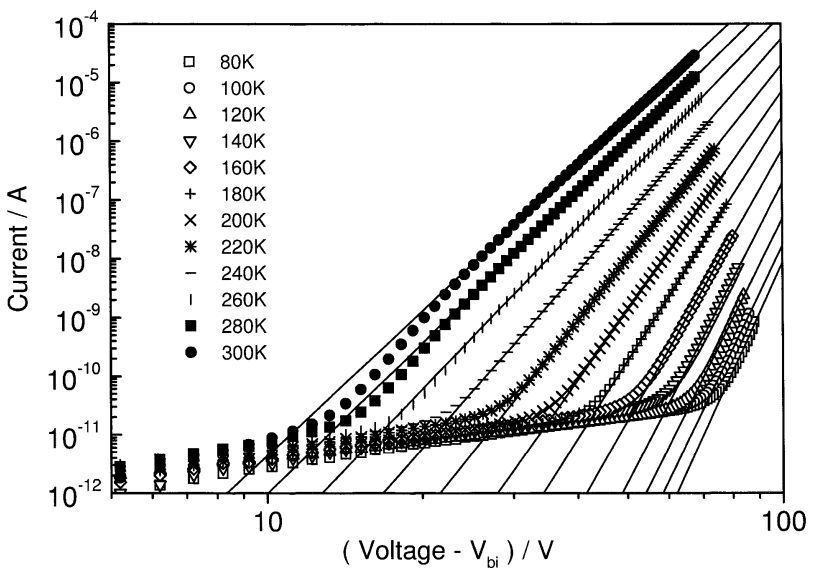

Fig. 4. Temperature-dependent $I-V$-characteristics of an ITO/Alq ${ }_{3}[550$ $\mathrm{nm}] /$ Ca device in double-logarithmic representation including the voltage correction due to the built-in potential. The straight lines indicate fits according to Eq. (1). At low currents a practically temperature-independent linear relationship between current and voltage can be observed which in our case is due to the finite insulation resistance of $10^{12} \Omega$ of our measurement setup.

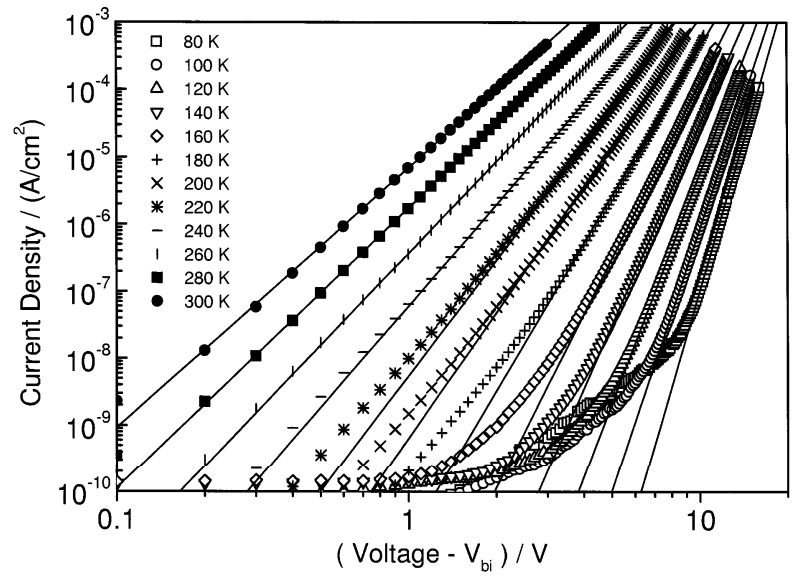

Fig. 5. Temperature-dependent $I-V$-characteristics of an ITO/TPD [60 $\mathrm{nm}] / \mathrm{Alq}_{3}[60 \mathrm{~nm}] / \mathrm{Ca}$ device in double-logarithmic representation with the voltage corrected by the built-in potential. The straight lines indicate fits according to Eq. (1).

carriers can be injected (ohmic contacts). In the following we will neglect the intrinsic charge carrier density, the field-dependence of the mobility and diffusion currents. Then for single layer devices with one type of charge carriers analytic solutions can be obtained for different energetic trap distributions. In the case of trap-free transport the current density is given by $j=(9 / 8) \varepsilon \mu V^{2} / d^{3}$ (also known as Child's law) with the dielectric constant $\varepsilon$ and the mobility $\mu$. Another important solution can be found for an exponential distribution of traps $N_{\mathrm{t}}(E)=$ $\left(H_{\mathrm{a}} / E_{\mathrm{t}}\right) \exp \left(-E / E_{\mathrm{t}}\right)$ [3] where $H_{\mathrm{a}}$ is the trap density, $E_{\mathrm{t}}$ the characteristic trap depth and $E$ the energy relative to the conduction or valence band:

$j \propto V^{m+1} / d^{2 m+1}$

where $m$ is given by $m=E_{\mathrm{t}} / k T>1$ and therefore only depends on the characteristic trap depth but not on the

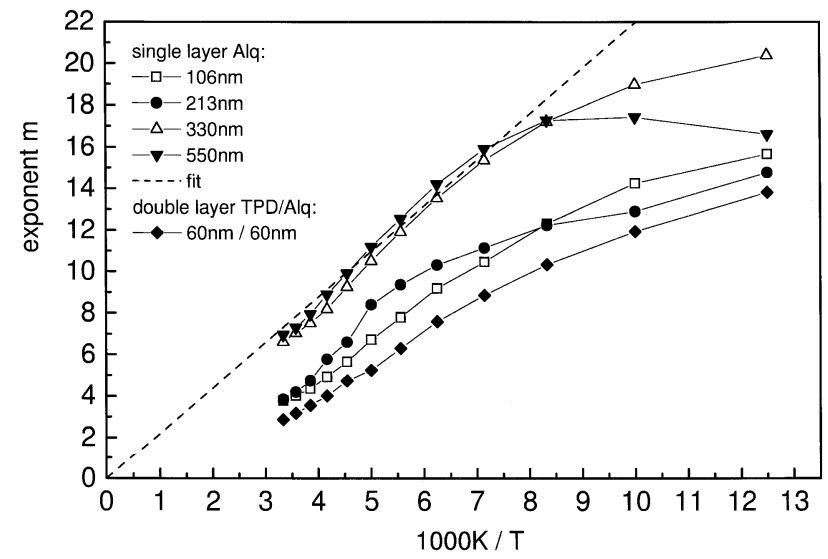

Fig. 6. Temperature dependence of the power law coefficient obtained by fitting the $I-V$-characteristics to Eq. (1) for single layer $\mathrm{Alq}_{3}$ devices with different organic layer thickness and for a TPD $/ \mathrm{Alq}_{3}$ hetero-structure. The fit to $m=E_{\mathrm{t}} / k T$ for the $550 \mathrm{~nm}$ thick device yields a characteristic trap depth of $E_{\mathrm{t}}=0.20 \mathrm{eV}$. 
device geometry (e.g., film thickness). Thus, a power law dependence of the $I-V$-characteristics following Eq. (1) can be regarded as indication for trap-limited SCLC. In the case of a non-negligible built-in potential, the voltage must of course be corrected by $V_{\text {bi }}$. Figs. $3-5$ show $I-V$-characteristics in a double logarithmic representation. In all cases the characteristics can be well described by temperature dependent power laws indicated by the straight lines, which is a hint to SCLC. The temperature dependence of the power law coefficient $m$ is shown in Fig. 6 for different $\mathrm{Alq}_{3}$ single layer devices and a TPD (60 $\mathrm{nm}) / \mathrm{Alq}_{3}(60 \mathrm{~nm})$ hetero-structure. For thick films and not too low temperatures the relationship $m \propto T^{-1}$ is valid and a characteristic trap depth of $0.2 \mathrm{eV}$ is obtained, which is comparable to a characteristic trap depth of $0.15 \mathrm{eV}$ observed earlier for the TPD/ $\mathrm{Alq}_{3}$ hetero-structure [4]. However, for thinner films a significant deviation can be seen leading to a thickness dependence of the power law coefficient and smaller values of $E_{\mathrm{t}}$. The values obtained for the hetero structure are similar to those obtained for the $106 \mathrm{~nm} \mathrm{Alq}{ }_{3}$ device, suggesting that the $I-V$-characteristics of the hetero structure is indeed dominated by the $\mathrm{Alq}_{3}$ layer. The observed deviations indicate that even for the single layer devices the simple trap-limited SCLC model is only an approximation. Possible reasons for the deviations are double injection, field-dependent mobility and the influence of residual injection barriers. However, to include such effects in more sophisticated models a numerical solution [5-8] of the corresponding differential equations is necessary, requiring also a better knowledge of the involved material parameters like trap density, charge carrier mobility or details of interfaces.

\section{Acknowledgements}

Financial support from the Bayerische Forschungs-stiftung, the Volkswagen Stiftung and the Sonderforschungsbereich 481 is gratefully acknowledged.

\section{References}

[1] M. Pope, H.P. Kallmann, P. Magnante, J. Chem. Phys. 37 (1963) 2042.

[2] C.W. Tang, S.A. Van Slyke, Appl. Phys. Lett. 51 (1987) 913.

[3] M.A. Lampert, P. Mark, Current Injections in Solids, Academic Press, New York, 1970.

[4] Z. Shen, P.E. Burrows, V. Bulovic, D.Z. Garbuzov, D.M. McCarty, M.E. Thompson, S.R. Forrest, Jpn. J. Appl. Phys., Part 235 (1996) L401.

[5] U. Wolf, V.I. Arkhipov, H. Bässler, Phys. Rev. B 59 (1999) 7507.

[6] P.W.M. Blom, M.J.M. de Jong, M.G. van Munster, Phys. Rev. B 55 (1997) R656.

[7] J. Yang, J. Shen, J. Appl. Phys. 85 (1999) 2699.

[8] A.J. Campbell, M.S. Weaver, D.G. Lidzey, D.D.C. Bradley, J. Appl. Phys. 84 (1998) 6737. 